\title{
Experimental Cannabinoid 2 Receptor-Mediated Immune Modulation in Sepsis
}

\author{
J. Sardinha, ${ }^{1}$ M. E. M. Kelly, ${ }^{1}$ J. Zhou, ${ }^{2,3}$ and C. Lehmann ${ }^{1,2,3}$ \\ ${ }^{1}$ Department of Pharmacology, Dalhousie University, Halifax, NS, Canada B3H 4R2 \\ ${ }^{2}$ Department of Anesthesia, Dalhousie University, Halifax, NS, Canada B3H 2 Y9 \\ ${ }^{3}$ Department of Microbiology \& Immunology, Dalhousie University, Halifax, NS, Canada B3H 4R2
}

Correspondence should be addressed to C. Lehmann; chlehmann@dal.ca

Received 7 November 2013; Revised 25 February 2014; Accepted 4 March 2014; Published 3 April 2014

Academic Editor: Magdalena Klink

Copyright (C) 2014 J. Sardinha et al. This is an open access article distributed under the Creative Commons Attribution License, which permits unrestricted use, distribution, and reproduction in any medium, provided the original work is properly cited.

\begin{abstract}
Sepsis is a complex condition that results from a dysregulated immune system in response to a systemic infection. Current treatments lack effectiveness in reducing the incidence and mortality associated with this disease. The endocannabinoid system offers great promise in managing sepsis pathogenesis due to its unique characteristics. The present study explored the effect of modulating the $\mathrm{CB}_{2}$ receptor pathway in an acute sepsis mouse model. Endotoxemia was induced by intravenous injection of lipopolysaccharide (LPS) in mice and intestinal microcirculation was assessed through intravital microscopy. We found that HU308 $\left(\mathrm{CB}_{2}\right.$ receptor agonist) reduced the number of adherent leukocytes in submucosal venules but did not restore muscular and mucosal villi FCD in endotoxemic mice. AM630 ( $\mathrm{CB}_{2}$ receptor antagonist) maintained the level of adherent leukocytes induced by LPS but further reduced muscular and mucosal villi FCD. URB597 (FAAH inhibitor) and JZL184 (MAGL inhibitor) both reduced the number of adherent leukocytes in submucosal venules but did not restore the mucosal villi FCD. Using various compounds we have shown different mechanisms of activating $\mathrm{CB}_{2}$ receptors to reduce leukocyte endothelial interactions in order to prevent further inflammatory damage during sepsis.
\end{abstract}

\section{Introduction}

Sepsis and septic shock are the leading causes of mortality in intensive care units worldwide [1]. Globally an estimated 19 million cases of sepsis occur per year, with one-third of the patients dying from the condition $[2,3]$. Sepsis is a complex immune syndrome characterized by an imbalance between pro- and anti-inflammatory mediators systemically released in high amounts (cytokine storm) in response to an infection $[4,5]$. During the early stages of sepsis, immune cells are often hyperactivated and may lose their ability to differentiate between infectious targets and healthy cells (horror autotoxicus). If left untreated, consequences of immune system dysregulation include impairment of circulatory function (septic shock), leading to subsequent poor tissue perfusion. Eventually, organ systems start failing from lack of nutrients leading to patient mortality [6]. Current treatments for sepsis include administration of antibiotics to fight the infection and fluid resuscitation and vasopressors to combat hypotension. However, there are no approved treatment options available that target the malfunctioning immune system [7].

The endocannabinoid system has recently emerged as a potential target in sepsis treatment [8]. This system is an endogenous signalling system that mediates a variety of physiological functions including modulation of the immune system. The endocannabinoid system is composed of endogenous ligands (endocannabinoids), cannabinoid receptors, and enzymes that synthesize and degrade endocannabinoids [9]. The two most well-known endocannabinoids are arachidonoylethanolamide (AEA; formerly known as anandamide) and 2-arachidonoylglycerol (2-AG) [10, 11]. These ligands activate a variety of receptors, but the two most important are the G-protein coupled cannabinoid $1\left(\mathrm{CB}_{1}\right)$ and cannabinoid $2\left(\mathrm{CB}_{2}\right)$ receptors [12]. $\mathrm{CB}_{1}$ receptors are found throughout the body, including in the central nervous system. These receptors mediate the psychotropic actions of the $\Delta^{9}$-THC, 
a phytocannabinoid constituent of Cannabis sativa. $\mathrm{CB}_{2}$ receptors are strongly expressed on the surface of immune cells $[13,14]$. It has been well documented that activation of the $\mathrm{CB}_{2}$ receptors causes an immunosuppressive response $[15,16]$. The effects of AEA and 2-AG are locally ablated by degradation through two enzymes, fatty acid amide hydrolase (FAAH) [17] and monoacylglycerol lipase (MAGL), respectively [18].

The aim of our study was to elucidate the potential role of $\mathrm{CB}_{2}$-mediated immune modulation in sepsis with a focus on the intestinal microcirculation that plays a critical role in the pathophysiology of sepsis $[19,20]$. During the initial stages of sepsis, a significant proinflammatory response occurs evidenced by marked increases in leukocyte endothelial interactions. We therefore investigated changes in leukocyte recruitment as well as functional capillary density in mice challenged with endotoxin as an acute experimental model of sepsis (endotoxemia). Previous studies have suggested a beneficial role of $\mathrm{CB}_{2}$ receptor activation in attenuating leukocyte endothelial interactions, as well as proinflammatory mediators [21-24]. These studies have shown that $\mathrm{CB}_{2}$ receptor activation can be beneficial in inflammatory states by reducing release of proinflammatory cytokines like TNF- $\alpha$, activation of endothelial cells, transmigration of inflammatory infiltrates, and reactive damage by oxidative stress and apoptosis. Our experiments examined the effects of $\mathrm{CB}_{2}$ receptor modulation on intestinal microcirculation by using $\mathrm{CB}_{2}$ agonists and antagonists and inhibitors of FAAH and MAGL.

\section{Methods}

2.1. Animals. Male C57BL/6 mice (6 weeks old; $20-30$ g) were purchased from Charles River Laboratories International Inc. (Wilmington, MS, USA). Animals were housed in ventilated rack cages and allowed to acclimatize for a week at the Carleton animal care facility of the Faculty of Medicine at Dalhousie University, Halifax, NS, Canada. Animals were kept on a standard 12-hour light/dark cycle, with standard room temperature $22^{\circ} \mathrm{C}$ and humidity 55\%-60\%. Animals were fed a standard diet of rodent chow and water ad libitum. This study was conducted in accordance with the guidelines and standards set forth by the Canadian Council on Animal Care and approved by the University Committee on Laboratory Animals at Dalhousie University.

\subsection{Endotoxemia. Animals were anesthetised with $90 \mathrm{mg} / \mathrm{kg}$} pentobarbital (Ceva Sante Animale, Montreal, QC, Canada) administered intraperitoneally (I.P.) and supplemented with $20 \mathrm{mg} / \mathrm{kg}$ pentobarbital intravenously (I.V.) during the experiment when needed. Animals breathed room air spontaneously, but oxygen was provided if breathing got laboured. The mouse was placed on a heating pad in supine position to maintain body temperature at $37^{\circ} \mathrm{C}\left(98.6^{\circ} \mathrm{F}\right)$ and a rectal temperature probe was used for the measurement. The left jugular vein was cannulated with polyethylene tubing (PE 10, Clay Adams, Sparks, MD, USA) for administration of fluids and drugs.
2.3. Experimental Groups. Six groups of animals were examined ( $N=3-5$ per group). Group 1 served as control (CON) group which only received saline $(0.9 \%$ sodium chloride, Hospira, Montreal, QC, Canada) at an equal volume of LPS as described below. All other groups received an I.V. dose of $5 \mathrm{mg} / \mathrm{kg}$ lipopolysaccharide (LPS, Escherichia coli, serotype O26:B6, Sigma-Aldrich, Oakville, ON, Canada). Group 2 received no further treatment besides LPS. Groups 3-6 received treatment compounds administered I.V. 15 minutes after administration of LPS. Group 3 received a $\mathrm{CB}_{2}$ receptor agonist, HU308 (2.5 mg/kg; Tocris Bioscience, Ellisville, MO, USA). Group 4 received a $\mathrm{CB}_{2}$ receptor antagonist/inverse agonist, AM630 $(2.5 \mathrm{mg} / \mathrm{kg}$; Tocris Bioscience, Ellisville, MO, USA). Group 5 received a FAAH inhibitor, URB597 (0.6 mg/kg; Tocris Bioscience, Ellisville, MO, USA). Group 6 received a MAGL inhibitor, JZL184 $(16 \mathrm{mg} / \mathrm{kg}$; Tocris Bioscience, Ellisville, MO, USA). All treatment compounds were dissolved in dimethyl sulfoxide (DMSO) and stock solutions were further diluted with saline to a final concentration of $10 \%$ (30\% for AM630). Pilot experiments did not show detrimental effects within the microcirculation using DMSO concentrations up to $50 \%$.

2.4. Intravital Microscopy. Intravital microscopy (IVM) of the terminal ileum was performed using an epifluorescent microscope (Leica DMLM, Wetzlar, Germany) with a mercury-arc light source (LEG EBQ 100; Carl Zeiss, Jena, Germany). Videos were recorded on a standard personal computer using IC capture software (v2.2, Imaging Source; Charlotte, NC, USA) and stored on external hard drives. To access the intestine, an abdominal midline incision was made. Using saline soaked cotton tipped applicators, a section of the terminal ileum was exposed and placed on a specially designed apparatus [25]. Using this apparatus, a liquid contact is made between the intestine and the cover slip. This technique minimizes pressure on the tissue in addition to the added benefit of constantly hydrating the intestine with thermostat controlled saline $\left(37^{\circ} \mathrm{C} / 98.6^{\circ} \mathrm{F}\right)$.

To observe leukocyte activation within the intestinal microvasculature, animals were intravenously administered $0.05 \%$ rhodamine $6 \mathrm{G}$-solution $(1.5 \mathrm{~mL} / \mathrm{kg}$, Sigma-Aldrich). Leukocyte adherence was observed in collecting venules (V1; $>50 \mu \mathrm{m}$ diameter) as well as postcapillary venules (V3; $<50 \mu \mathrm{m}$ diameter). Leukocytes that remained immobile on the endothelium for 30 seconds qualified as adherent leukocytes, while all nonadherent leukocytes patrolling past a designated point across the vessel were quantified as rolling leukocytes. This data allowed us to estimate the number of adherent leukocytes (cells $/ \mathrm{mm}^{2}$ ), as well as the number of rolling leukocytes (cells/minute), along the intestinal endothelium. To observe microvascular integrity, animals were intravenously administered 5\% fluorescein isothiocyanate- (FITC-) tagged albumin ( $1 \mathrm{~mL} / \mathrm{kg}$; SigmaAldrich). Capillary perfusion in the muscle layers and the mucosal villi of the intestine was observed. To visualize the mucosal villi, the intestinal surface was cauterized and cut to expose the lumen. The length of perfused capillaries was measured in a defined area and used to calculate 
the functional capillary density (FCD $\left.\mu \mathrm{m} / \mu \mathrm{m}^{2}\right)$. Six visual fields of each vascular type were recorded for 30 seconds each.

2.5. Statistical Analysis. Results were analyzed by using the software Prism 5 (GraphPad Software, La Jolla, CA, USA). All data were analysed using a one-way ANOVA with a post hoc Newman-Keuls correction. All data are expressed as means \pm standard deviation (SD). Significance was set at $P<0.05$.

\section{Results}

3.1. Leukocyte Adhesion. Endotoxin challenge significantly $(P<0.05)$ increased the number of adherent leukocytes in V1 and V3 venules compared to controls (Figures 1(a) and 1(b)). They showed a 100-fold increase in V1 venules and a 10 -fold increase in $\mathrm{V} 3$ venules.

Administration of HU308, URB597, or JZL184 after LPS challenge significantly $(P<0.05)$ reduced the number of adherent leukocytes in V1 and V3 venules in comparison to untreated LPS animals (Figures 1(a) and 1(b). These treatments following LPS challenge reduced the number of adherent leukocytes to the same levels as the non-LPSchallenged control group in V1 (Figure 1(a); $P>0.05$ ) but not in V3 venules (Figure 1(b); $P<0.05$ ). Administration of AM630 after LPS challenge showed no significant $(P>0.05)$ difference in leukocyte adherence to LPS group in V1 and V3 venules (Figures 1(a) and 1(b)).

3.2. Leukocyte Rolling. In comparison to controls, LPSchallenged animals showed a significant $(P<0.05)$ reduction in the number of rolling leukocytes for both V1 and V3 venules (Figures 2(a) and 2(b)). Administration of either HU308, AM630, URB597, or JZL184 after LPS challenge did not change the number of rolling leukocytes in comparison to LPS alone group in V1 venules (Figure 2(a)) and V3 venules (Figure 2(b)).

3.3. Functional Capillary Density (FCD). Muscular functional capillary density showed no significant differences between controls and LPS (Figure 3). LPS + AM630 showed a significant reduction in muscle layer FCD compared to control. All other treatment groups showed no significant differences in muscular FCD when compared to controls or LPS. Mucosal FCD showed a significant reduction for all groups when compared to controls (Figure 4).

\section{Discussion}

In our study we compared for the first time the impact of various approaches to activate the $\mathrm{CB}_{2}$ receptor pathway in regard to leukocyte activation and functional capillary density within the intestinal microcirculation, which is a critical component in sepsis pathophysiology. We demonstrated the benefit of the $\mathrm{CB}_{2}$ receptor agonist, HU308, in reducing LPSinduced leukocyte recruitment. Furthermore, an alternate mechanism of activating the $\mathrm{CB}_{2}$ pathway through the enzyme inhibitors URB597 (FAAH) and JZL184 (MAGL) produced similar results. In contrast, blocking $\mathrm{CB}_{2}$ receptors by $\mathrm{CB}_{2}$ receptor antagonist $\mathrm{AM} 630$ caused $\mathrm{FCD}$ reduction in intestinal musculature.

Our endotoxemic model produced a robust immune response by increasing the number of adherent leukocytes within V1 and V3 venules and reducing the number of rolling leukocytes. LPS also affected capillary function as the FCD for the mucosal villi was reduced, but muscular FCD was not significantly lowered indicating an initiation of vascular damage. Our first therapeutic approach examined the effects of $\mathrm{a} \mathrm{CB}_{2}$ receptor agonist HU308 on the intestinal microcirculation. Studies using $\mathrm{CB}_{2}$ agonists have indicated protective effects against inflammatory damage in various organs $[14,23]$. They have shown reduction in expression of adhesion molecules such as intercellular adhesion molecule (ICAM) and vascular cell adhesion molecule (VCAM) [21, 24], reduction in levels of proinflammatory cytokines such as tumor necrosis factor- $\alpha$ (TNF- $\alpha)[21,26]$, and decreased neutrophil infiltration but increased neutrophil activation [27]. Our results showed that HU308 was able to reduce the number of LPS-induced adherent leukocytes in V1 and V3 venules; however, the number of rolling leukocytes did not return to the levels seen in controls. These results suggest that HU308 suppresses leukocyte recruitment alluding to the lack of rolling leukocytes observed. HU308 treatment showed no improvement on mucosal villi FCD, indicating that this treatment did not have a sufficient impact on the proinflammatory mediators in preventing vascular damage. In general, HU308 administration showed some benefit after LPS administration by reducing the number of adherent leukocytes in both V1 and V3 venules, adding some support to other studies using $\mathrm{CB}_{2}$ agonist administration which show a reduction in leukocyte chemotaxis, endothelial interaction and transmigration, and release of proinflammatory mediators in experimental models of endotoxemia [26-29].

In order to further verify our findings that $\mathrm{CB}_{2}$ receptor activation through HU308 reduces LPS-induced microcirculatory damage, we blocked $\mathrm{CB}_{2}$ receptor activation with a $\mathrm{CB}_{2}$ receptor antagonist/inverse agonist AM630. Other studies have shown exacerbated inflammatory damage in their disease model with the use of AM630 [30] and neutralized therapeutic effects of AM630 in combination with a $\mathrm{CB}_{2}$ ligand $[31,32]$. Our results correspond with the expected outcomes as AM630 maintained the number of adherent leukocytes induced by LPS. It is possible that maximal leukocyte recruitment may have been achieved after LPS challenge and therefore administration of AM630 following LPS could not further elevate leukocyte adherence. Furthermore, mucosal villi FCD was reduced and AM630 was the only group showing reduction in the muscular layers. It is possible that inflammatory processes were elevated beyond LPS-induced levels, indicated by a reduction in muscular FCD, even though exacerbated inflammation was not evident through leukocyte endothelial interactions.

Another therapeutic approach to activate the $\mathrm{CB}_{2}$ pathway is through inhibition of endocannabinoid hydrolysing enzymes. Inhibiting enzymes that degrade endocannabinoids can cause elevated levels of the endogenous compounds AEA and 2-AG, resulting in prolonged stimulation of the $\mathrm{CB}_{2}$ receptors. Using various synthetic compounds and $\mathrm{FAAH}$ 


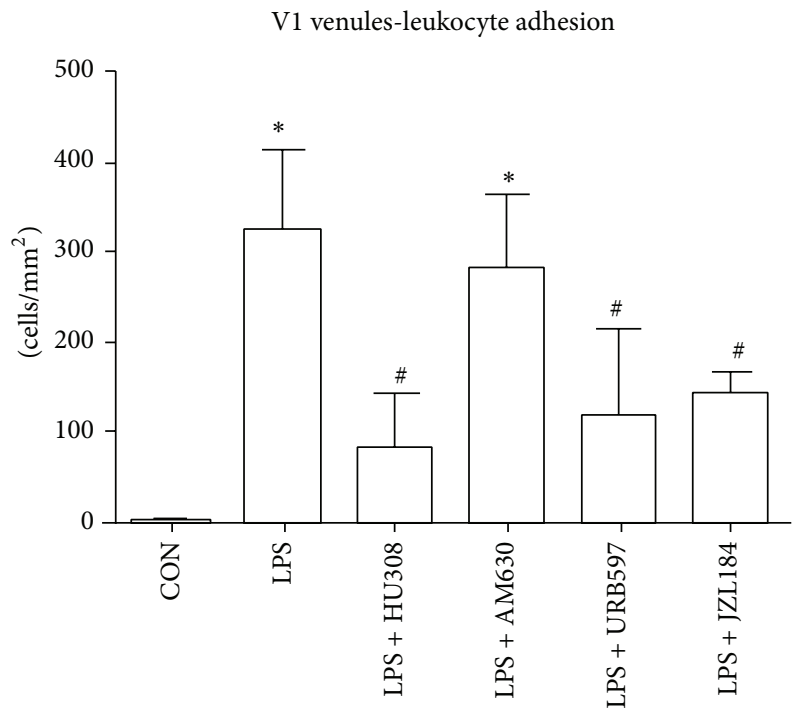

(a)
V3 venules-leukocyte adhesion

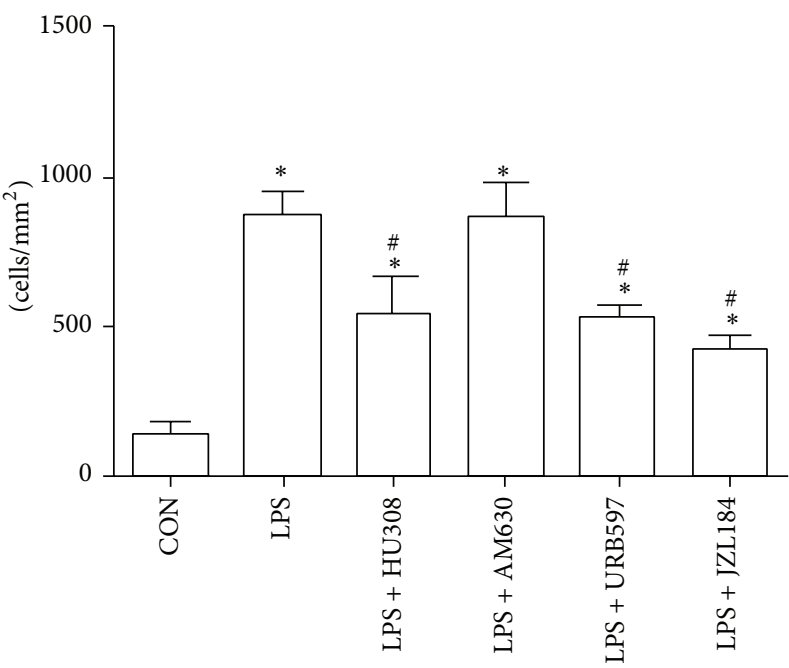

(b)

FIGURE 1: (a) Adherent leukocytes in collecting venules (V1; $>50 \mu \mathrm{m}$ vessel diameter). (b) Adherent leukocytes in postcapillary venules (V3; $<50 \mu \mathrm{m}$ vessel diameter). Control group (CON); endotoxemia group LPS ( $5 \mathrm{mg} / \mathrm{kg}) ; \mathrm{LPS}+\mathrm{HU} 308(2.5 \mathrm{mg} / \mathrm{kg})$, a CB ${ }_{2}$ receptor agonist; LPS + AM630 (2.5 mg/kg), a CB 2 receptor antagonist/inverse agonist; LPS + URB597 (0.6 mg/kg), a FAAH inhibitor; and LPS + JZL184 (16 mg/kg) a MAGL inhibitor. In every animal six V1 venules and six V3 venules were analyzed ( $n=3-5$ mice/group). Data presented as mean \pm standard deviation. ${ }^{*} P<0.05$ versus control. ${ }^{\#} P<0.05$ versus LPS.

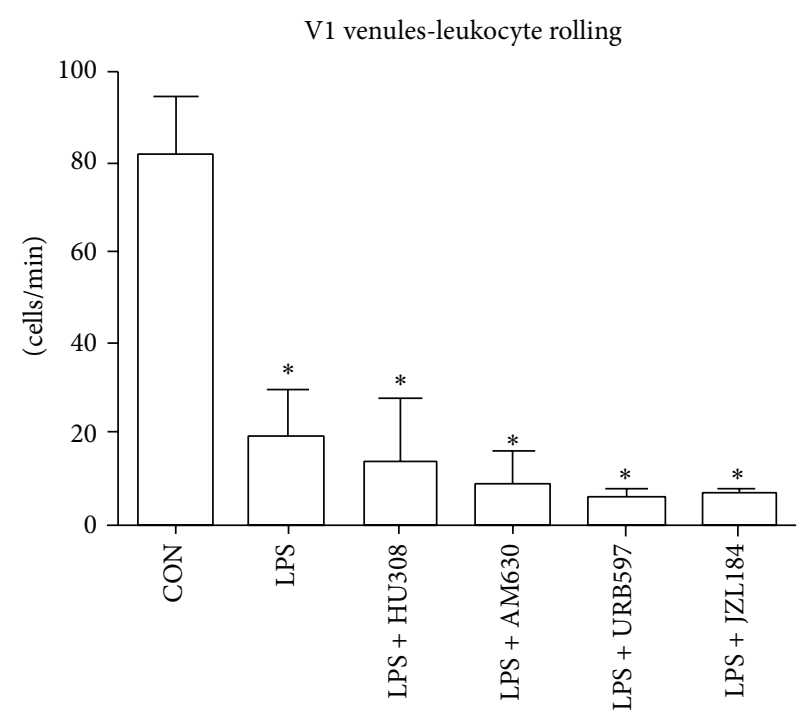

(a)

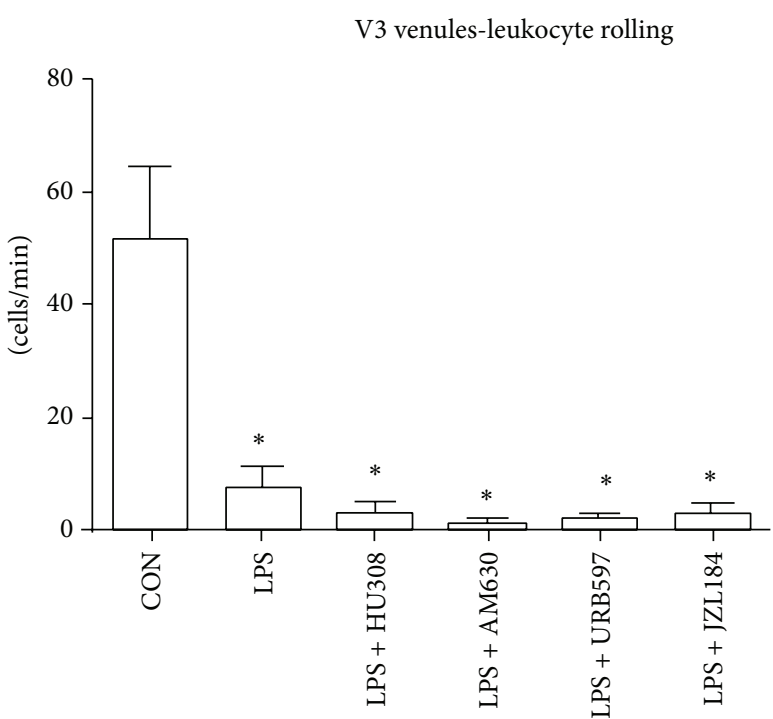

(b)

FIGURE 2: (a) Rolling leukocytes along collecting venules (V1; $>50 \mu \mathrm{m}$ vessel diameter). (b) Rolling leukocytes along postcapillary venules (V3; $<50 \mu \mathrm{m}$ vessel diameter). Control group (CON); endotoxemia group LPS ( $5 \mathrm{mg} / \mathrm{kg}) ; \mathrm{LPS}+\mathrm{HU} 308(2.5 \mathrm{mg} / \mathrm{kg})$, a CB 2 receptor agonist; LPS + AM630 (2.5 mg/kg), a CB 2 receptor antagonist/inverse agonist; LPS + URB597 (0.6 mg/kg), a FAAH inhibitor; and LPS + JZL184 (16 mg/kg), a MAGL inhibitor. In every animal six V1 venules and six V3 venules were analyzed ( $n=3-5$ mice/group). Data presented as mean \pm standard deviation. ${ }^{*} P<0.05$ versus control.

deficient mice, researchers have shown that FAAH inhibition reduced hydrolysis rates for AEA, reduced pain sensitivity and analgesia, and reduced inflammation, all indicative of elevated AEA levels and cannabinoid receptor activation [33-36]. Our results with an irreversible FAAH inhibitor
URB597 indicated analogous findings to HU308 administration: leukocyte endothelial interactions were reduced in both submucosal V1 and V3 venules, and FCD was not improved in muscular and mucosal villi. Previous studies in our laboratory using URB597 in a rat model of endotoxemia showed 


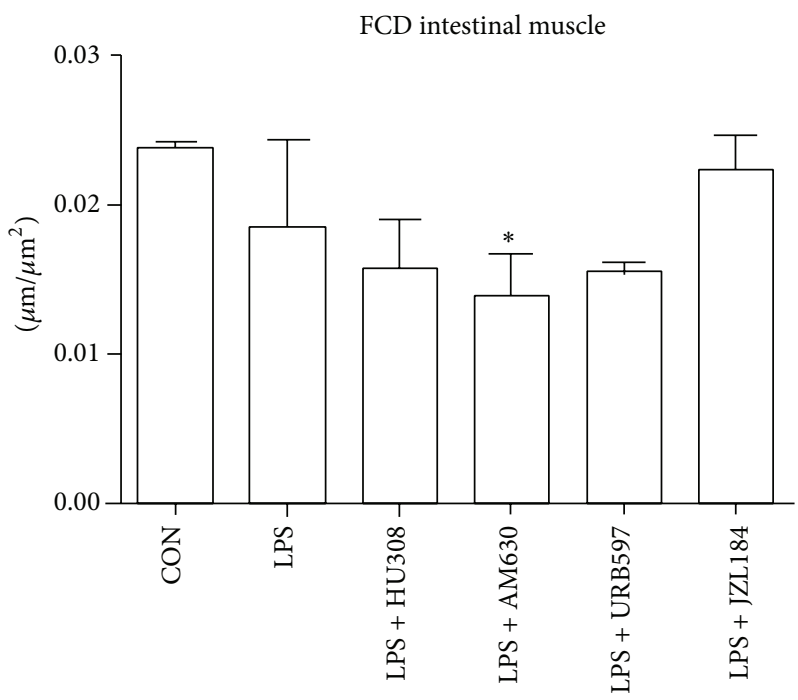

FIGURE 3: Functional capillary density (FCD) as a measure of capillary perfusion within the muscle layers of the intestine. Calculated as total length of capillaries with erythrocyte perfusion within a predetermined rectangular field. Control group (CON); endotoxemia group LPS $(5 \mathrm{mg} / \mathrm{kg}) ; \mathrm{LPS}+\mathrm{HU} 308(2.5 \mathrm{mg} / \mathrm{kg}), \mathrm{a} \mathrm{CB}_{2}$ receptor agonist; LPS + AM630 (2.5 mg/kg), a CB ${ }_{2}$ receptor antagonist/inverse agonist; LPS + URB597 $(0.6 \mathrm{mg} / \mathrm{kg})$, a FAAH inhibitor; and LPS + JZL184 $(16 \mathrm{mg} / \mathrm{kg})$, a MAGL inhibitor. In every animal six intestinal muscle regions were analyzed ( $n=3-5$ mice/group). Data presented as mean \pm standard deviation. ${ }^{*} P<0.05$ versus control.

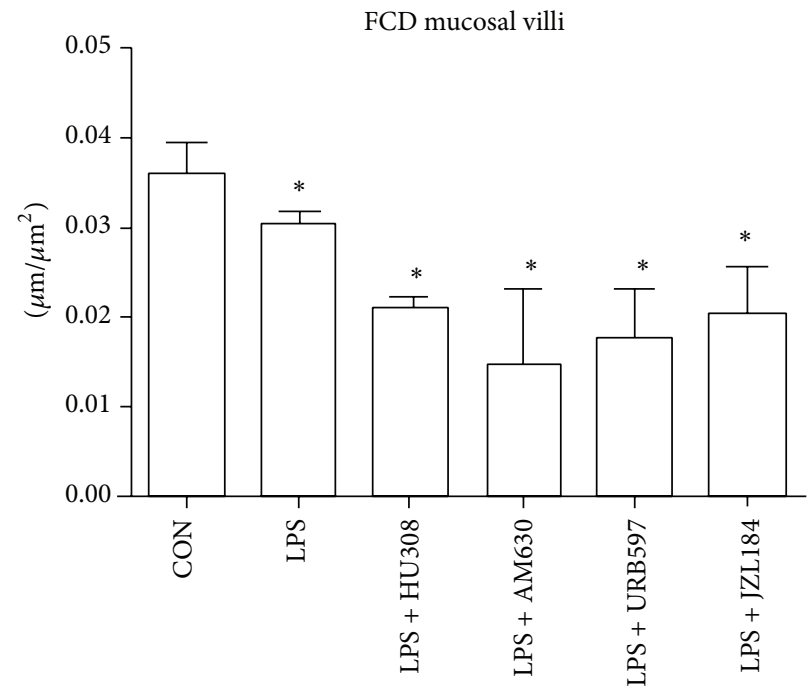

FIGURE 4: Functional capillary density (FCD) as a measure of capillary perfusion within mucosal villi. Calculated as total length of capillaries with erythrocyte perfusion within a predetermined rectangular field. Control group (CON); endotoxemia group LPS (5 mg/kg); LPS + HU308 $(2.5 \mathrm{mg} / \mathrm{kg})$, a $\mathrm{CB}_{2}$ receptor agonist; LPS + AM630 (2.5 mg/kg), a $\mathrm{CB}_{2}$ receptor antagonist/inverse agonist; LPS + URB597 (0.6 mg/kg), a FAAH inhibitor; LPS + JZL184 (16 mg/kg), a MAGL inhibitor. In every animal six mucosal regions were analyzed ( $n=3-5 \mathrm{mice} / \mathrm{group})$. Data presented as mean \pm standard deviation. ${ }^{*} P<0.05$ versus control.

comparable results with the exception of a therapeutic benefit seen in elevating mucosal villi and circular muscle FCD [32].

The most common MAGL inhibitor used experimentally in vivo is JZL184 [37, 38]. A recent study investigated MAGL knockout (K.O.) mice in a hepatic injury model [39]. The results showed wild-type mice given JZL184 and MAGL K.O. mice were protected from hepatic ischemia/reperfusion injury through increased $\mathrm{CB}_{2}$ signalling. JZL184 suppressed the oxidative stress and inflammation that resulted from ischemia/reperfusion by increasing endocannabinoid levels through MAGL inhibition. Our results with JZL184 showed similar findings with reduced inflammation indicated by less adherent leukocytes in both V1 and V3 venules. As seen with URB597, JZL184 administration showed no difference in muscular FCD to the LPS group, while mucosal villi FCD did not show significant improvement compared to LPS administration alone.

Our data indicate comparable results between HU308, URB597, and JZL184; however, we favour further testing with enzyme inhibitors over synthetic agonists for the following 
reasons. One advantage of using enzyme inhibitors is that potential side effects of synthetic agonist administration, for example, receptor desensitization or tachyphylaxis, can be minimized. Furthermore, hydrolysing enzymes work at local sites of endogenous cannabinoids production; therefore, a more precise activation of target receptors is possible due to localized increases in endocannabinoid levels. Disadvantages of using (unspecific) $\mathrm{CB}_{2}$ receptor agonists systemically are the potential activation of off-target receptors, for example, GPR55 [40]. With systemically elevated levels of endocannabinoids, alternative pathways like the eicosanoid pathway may be activated [41]. Arachidonic acid which is a metabolite of $2-\mathrm{AG}$ is also a precursor molecule for prostaglandins and leukotrienes. In this study the use of JLZ184, a MAGL inhibitor, prevents 2-AG metabolism, thereby minimizing availability of arachidonic acid to be converted to leukotrienes and prostanoids. However, as an alternative, cyclooxygenases can oxygenate endocannabinoids which can then be hydrolyzed to prostaglandins. Endocannabinoid levels are relatively low even when enzyme inhibitors are employed, limiting the effect of the eicosanoid pathway in our study [41]. Another possible drawback of elevating endocannabinoid levels is their effects on off-target receptors like $\mathrm{CB}_{1}$ and $\mathrm{GPR} 55$. However, $\mathrm{CB}_{1}$-mediated effects, if present, would potentially increase leukocyte activation since inhibition of the $\mathrm{CB}_{1}$ pathway was shown by our group to reduce leukocyte adhesion to the endothelium in experimental endotoxemia [42]. GPR55 is highly expressed not only in the brain, but also in the gastrointestinal tract $[43,44]$. Unfortunately, the role of GPR55 in intestinal inflammation has not yet been fully elucidated.

Although our acute endotoxin model was quite effective in producing an inflammatory response, this model has limited clinical relevance due to lack of pathogens in the body and lack of the multiphasic immune states evident during sepsis pathogenesis. Clinical sepsis in patients is usually triggered by an infecting pathogen; therefore, due to the lack of an active pathogen in our model, our results should be interpreted with some caution when translating to clinical relevance. More clinically relevant models of sepsis induce abdominal peritonitis through fecal translocation from the intestine $[27,45]$. However, controversy still exists on the benefit of the $\mathrm{CB}_{2}$ pathway in these models [8]. Sepsis pathophysiology is quite complex in clinical settings, with time-dependent changes of the status of the immune system. Our study examined the immune state two hours after endotoxin administration thereby limiting our model to the initial proinflammatory phase of sepsis pathogenesis. Another limitation to our study is the possibility of a dosedependent effect with our compounds. All our compound doses were based on previous dose response studies conducted in either rats or mice $[29,32,46]$.

In conclusion, our results support the concept of targeting the $\mathrm{CB}_{2}$ pathway in sepsis to modulate the acute phase response of the immune system. We demonstrated the therapeutic benefit of the $\mathrm{CB}_{2}$ receptor agonist HU308 in reducing LPS-induced leukocyte recruitment within the intestinal microcirculation. Furthermore, an alternate mechanism of activating the $\mathrm{CB}_{2}$ pathway through the enzyme inhibitors URB597 and JZL184 produced similar results. In contrast, blocking $\mathrm{CB}_{2}$ receptors with $\mathrm{AM} 630$ caused additional FCD reduction in intestinal musculature. These results support the benefit of modulating the $\mathrm{CB}_{2}$ pathway in sepsis pathogenesis, warranting further investigation into this pathway in order to develop effective therapeutics.

\section{Conflict of Interests}

All authors have declared that there is no conflict of interests.

\section{References}

[1] J.-L. Vincent, J. Rello, J. Marshall et al., "International study of the prevalence and outcomes of infection in intensive care units," The Journal of the American Medical Association, vol. 302, no. 21, pp. 2323-2329, 2009.

[2] N. K. Adhikari, R. A. Fowler, S. Bhagwanjee, and G. D. Rubenfeld, "Critical care and the global burden of critical illness in adults," The Lancet, vol. 376, no. 9749, pp. 1339-1346, 2010.

[3] Global Sepsis Aliance, "World Sepsis Day," 2012, http://www .world-sepsis-day.org.

[4] R. M. Kleinpell, B. T. Graves, and M. H. Ackerman, "Incidence, pathogenesis, and management of sepsis: an overview," AACN Advanced Critical Care, vol. 17, no. 4, pp. 385-393, 2006.

[5] R. C. Bone, "The pathogenesis of sepsis," Annals of Internal Medicine, vol. 115, no. 6, pp. 457-469, 1991.

[6] J. Cohen, "The immunopathogenesis of sepsis," Nature, vol. 420, no. 6917, pp. 885-891, 2002.

[7] R. P. Dellinger, M. M. Levy, A. Rhodes et al., "Surviving Sepsis Campaign: international guidelines for management of severe sepsis and septic shock, 2012," Intensive Care Medicine, vol. 39, no. 2, pp. 165-228, 2013.

[8] J. Sardinha, C. Lehmann, and M. Kianian, "Targeting the endocannabinoid system to treat sepsis," Signa Vitae, vol. 8, no. 1, pp. 9-14, 2013.

[9] R. G. Pertwee, "Cannabinoid pharmacology: the first 66 years," British Journal of Pharmacology, vol. 147, no. 1, pp. S163-S171, 2006.

[10] W. A. Devane, L. Hanus, A. Breuer et al., "Isolation and structure of a brain constituent that binds to the cannabinoid receptor," Science, vol. 258, no. 5090, pp. 1946-1949, 1992.

[11] R. Mechoulam, S. Ben-Shabat, L. Hanus et al., "Identification of an endogenous 2-monoglyceride, present in canine gut, that binds to cannabinoid receptors," Biochemical Pharmacology, vol. 50, no. 1, pp. 83-90, 1995.

[12] R. G. Pertwee and R. A. Ross, "Cannabinoid receptors and their ligands," Prostaglandins, Leukotrienes and Essential Fatty Acids, vol. 66, no. 2-3, pp. 101-121, 2002.

[13] S. Munro, K. L. Thomas, and M. Abu-Shaar, "Molecular characterization of a peripheral receptor for cannabinoids," Nature, vol. 365, no. 6441, pp. 61-65, 1993.

[14] P. Pacher and R. Mechoulam, "Is lipid signaling through cannabinoid 2 receptors part of a protective system?" Progress in Lipid Research, vol. 50, no. 2, pp. 193-211, 2011.

[15] S. Chuchawankul, M. Shima, N. E. Buckley, C. B. Hartmann, and K. L. McCoy, "Role of cannabinoid receptors in inhibiting macrophage costimulatory activity," International Immunopharmacology, vol. 4, no. 2, pp. 265-278, 2004. 
[16] J. Ehrhart, D. Obregon, T. Mori et al., "Stimulation of cannabinoid receptor $2\left(\mathrm{CB}_{2}\right)$ suppresses microglial activation," Journal of Neuroinflammation, vol. 2, article 29, 2005.

[17] B. F. Cravatt, D. K. Giang, S. P. Mayfield, D. L. Boger, R. A. Lerner, and N. B. Gilula, "Molecular characterization of an enzyme that degrades neuromodulatory fatty-acid amides," Nature, vol. 384, no. 6604, pp. 83-87, 1996.

[18] K. Ahn, M. K. McKinney, and B. F. Cravatt, "Enzymatic pathways that regulate endocannabinoid signaling in the nervous system," Chemical Reviews, vol. 108, no. 5, pp. 1687-1707, 2008.

[19] C. Ince, "The microcirculation is the motor of sepsis," Critical Care, vol. 9, supplement 4, pp. S13-S19, 2005.

[20] A. Spanos, S. Jhanji, A. Vivian-Smith, T. Harris, and R. M. Pearse, "Early microvascular changes in sepsis and severe sepsis," Shock, vol. 33, no. 4, pp. 387-391, 2010.

[21] M. Rajesh, P. Mukhopadhyay, S. Bátkai et al., " $\mathrm{CB}_{2}$-receptor stimulation attenuates TNF- $\alpha$-induced human endothelial cell activation, transendothelial migration of monocytes, and monocyte-endothelial adhesion," American Journal of Physiology: Heart and Circulatory Physiology, vol. 293, no. 4, pp. H2210-H2218, 2007.

[22] M. Rajesh, H. Pan, P. Mukhopadhyay et al., "Pivotal Advance: cannabinoid-2 receptor agonist HU-308 protects against hepatic ischemia/reperfusion injury by attenuating oxidative stress, inflammatory response, and apoptosis," Journal of Leukocyte Biology, vol. 82, no. 6, pp. 1382-1389, 2007.

[23] T. W. Klein, "Cannabinoid-based drugs as anti-inflammatory therapeutics," Nature Reviews Immunology, vol. 5, no. 5, pp. 400-411, 2005.

[24] S. H. Ramirez, J. Haskó, A. Skuba et al., "Activation of cannabinoid receptor 2 attenuates leukocyte-endothelial cell interactions and blood-brain barrier dysfunction under inflammatory conditions," The Journal of Neuroscience, vol. 32, no. 12, pp. 4004-4016, 2012.

[25] C. Lehmann, F. Götz, L. Schuster, and J. Zhou, "Improved setup for intestinal intravital microscopy in mice-the, 'floating table," Minerva Anestesiologica, vol. 79, no. 1, pp. 102-103, 2013.

[26] H. Gui, Y. Sun, Z. M. Luo, D. F. Su, S. M. Dai, and X. Liu, "Cannabinoid receptor 2 protects against acute experimental sepsis in mice," Mediators of Inflammation, vol. 2013, Article ID 741303, 10 pages, 2013.

[27] J. Tschöp, K. R. Kasten, R. Nogueiras et al., "The cannabinoid receptor 2 is critical for the host response to sepsis," The Journal of Immunology, vol. 183, no. 1, pp. 499-505, 2009.

[28] S. R. Smith, C. Terminelli, and G. Denhardt, "Effects of cannabinoid receptor agonist and antagonist ligands on production of inflammatory cytokines and anti-inflammatory interleukin-10 in endotoxemic mice," Journal of Pharmacology and Experimental Therapeutics, vol. 293, no. 1, pp. 136-150, 2000.

[29] C. Lehmann, M. Kianian, J. Zhou et al., "Cannabinoid receptor 2 activation reduces intestinal leukocyte recruitment and systemic inflammatory mediator release in acute experimental sepsis," Critical Care, vol. 16, no. 2, article R47, 2012.

[30] M. A. Storr, C. M. Keenan, H. Zhang, K. D. Patel, A. Makriyannis, and K. A. Sharkey, "Activation of the cannabinoid 2 receptor $\left(\mathrm{CB}_{2}\right)$ protects against experimental colitis," Inflammatory Bowel Diseases, vol. 15, no. 11, pp. 1678-1685, 2009.

[31] A. Quartilho, H. P. Mata, M. M. Ibrahim et al., "Inhibition of inflammatory hyperalgesia by activation of peripheral $\mathrm{CB}_{2}$ cannabinoid receptors," Anesthesiology, vol. 99, no. 4, pp. 955960, 2003.
[32] M. Kianian, N. A. Al-Banna, M. E. Kelly, and C. Lehmann, "Inhibition of endocannabinoid degradation in experimental endotoxemia reduces leukocyte adhesion and improves capillary perfusion in the gut," Journal of Basic and Clinical Physiology and Pharmacology, vol. 24, no. 1, pp. 27-33, 2013.

[33] A. G. Hohmann, R. L. Suplita, N. M. Bolton et al., "An endocannabinoid mechanism for stress-induced analgesia," Nature, vol. 435, no. 7045, pp. 1108-1112, 2005.

[34] S. Holt, F. Comelli, B. Costa, and C. J. Fowler, "Inhibitors of fatty acid amide hydrolase reduce carrageenan-induced hind paw inflammation in pentobarbital-treated mice: comparison with indomethacin and possible involvement of cannabinoid receptors," British Journal of Pharmacology, vol. 146, no. 3, pp. 467-476, 2005.

[35] A. H. Lichtman, D. Leung, C. C. Shelton et al., "Reversible inhibitors of fatty acid amide hydrolase that promote analgesia: evidence for an unprecedented combination of potency and selectivity," Journal of Pharmacology and Experimental Therapeutics, vol. 311, no. 2, pp. 441-448, 2004.

[36] B. F. Cravatt, K. Demarest, M. P. Patricelli et al., "Supersensitivity to anandamide and enhanced endogenous cannabinoid signaling in mice lacking fatty acid amide hydrolase," Proceedings of the National Academy of Sciences of the United States of America, vol. 98, no. 16, pp. 9371-9376, 2001.

[37] M. Alhouayek, D. M. Lambert, N. M. Delzenne, P. D. Cani, and G. G. Muccioli, "Increasing endogenous 2-arachidonoylglycerol levels counteracts colitis and related systemic inflammation," The FASEB Journal, vol. 25, no. 8, pp. 2711-2721, 2011.

[38] S. M. Saario and J. T. Laitinen, "Therapeutic potential of endocannabinoid-hydrolysing enzyme inhibitors," Basic \& Clinical Pharmacology \& Toxicology, vol. 101, no. 5, pp. 287-293, 2007.

[39] Z. Cao, M. M. Mulvihill, P. Mukhopadhyay et al., "Monoacylglycerol lipase controls endocannabinoid and eicosanoid signaling and hepatic injury in mice," Gastroenterology, vol. 144, no. 4, pp. 808.e15-817.e15, 2013.

[40] R. G. Pertwee, "GPR55: a new member of the cannabinoid receptor clan?” British Journal of Pharmacology, vol. 152, no. 7, pp. 984-986, 2007.

[41] C. A. Rouzer and L. J. Marnett, "Endocannabinoid oxygenation by cyclooxygenases, lipoxygenases, and cytochromes P450: cross-talk between the eicosanoid and endocannabinoid signaling pathways," Chemical Reviews, vol. 111, no. 10, pp. 5899-5921, 2011.

[42] M. Kianian, M. E. Kelly, J. Zhou et al., "Cannabinoid receptor 1 inhibition improves the intestinal microcirculation," Clinical Hemorheology and Microcirculation, 2013.

[43] E. Ryberg, N. Larsson, S. Sjögren et al., "The orphan receptor GPR55 is a novel cannabinoid receptor," British Journal of Pharmacology, vol. 152, no. 7, pp. 1092-1101, 2007.

[44] R. Schicho and M. Storr, "A potential role for GPR55 in gastrointestinal functions," Current Opinion in Pharmacology, vol. 12, no. 6, pp. 653-658, 2012.

[45] B. Csóka, Z. H. Németh, P. Mukhopadhyay et al., " $\mathrm{CB}_{2}$ cannabinoid receptors contribute to bacterial invasion and mortality in polymicrobial sepsis," PLoS ONE, vol. 4, no. 7, article e6409, 2009.

[46] J. Z. Long, W. Li, L. Booker et al., "Selective blockade of 2arachidonoylglycerol hydrolysis produces cannabinoid behavioral effects," Nature Chemical Biology, vol. 5, no. 1, pp. 37-44, 2009. 


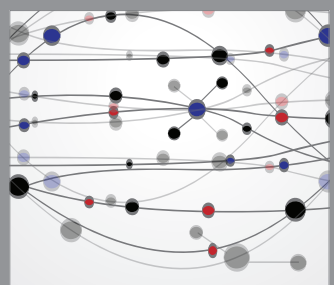

The Scientific World Journal
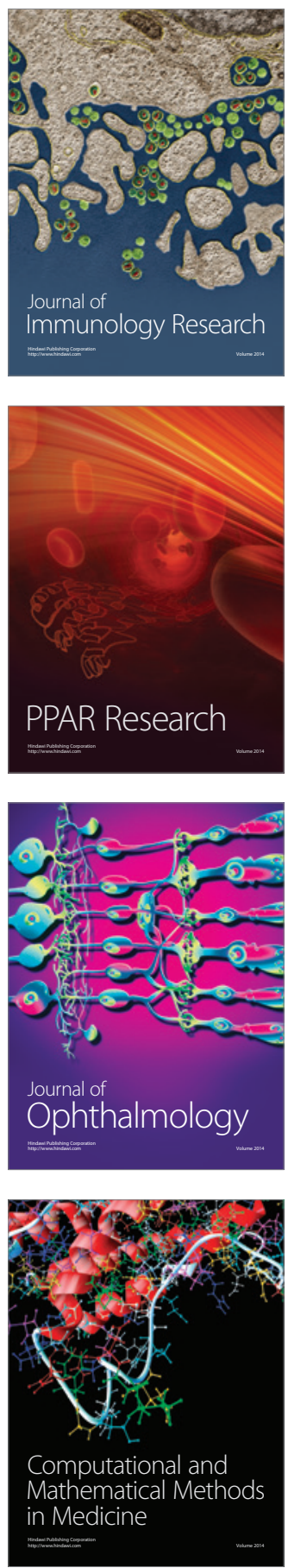

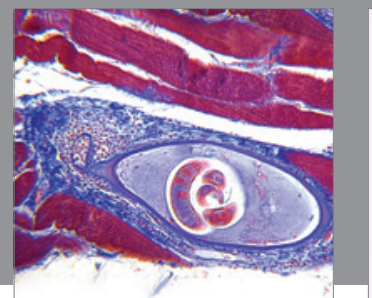

Gastroenterology

Research and Practice
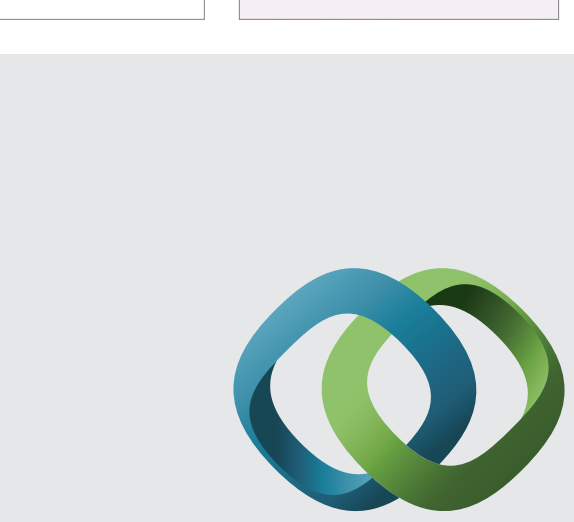

\section{Hindawi}

Submit your manuscripts at

http://www.hindawi.com
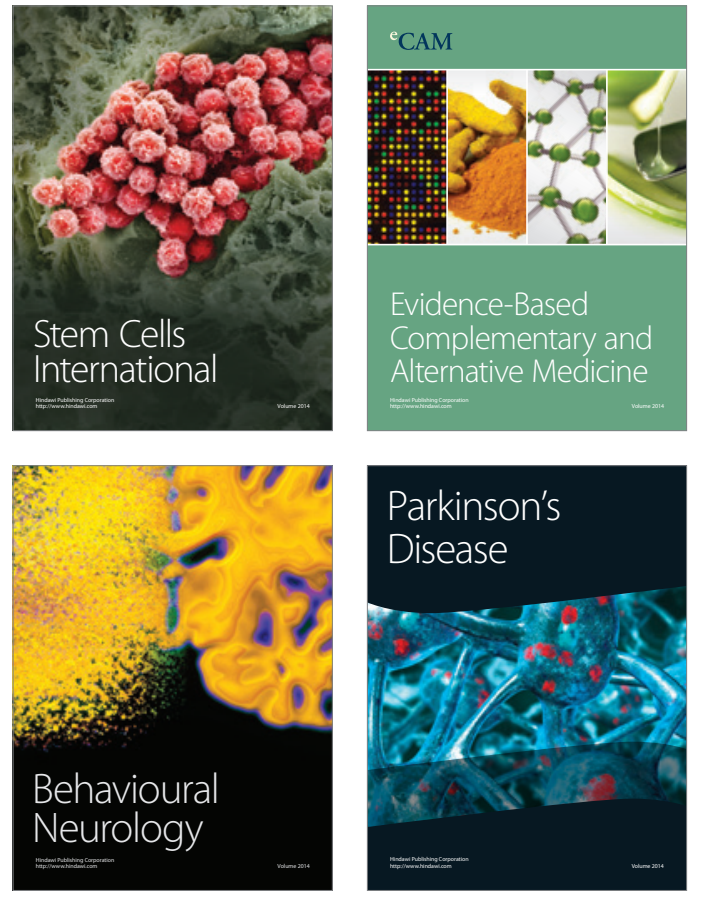
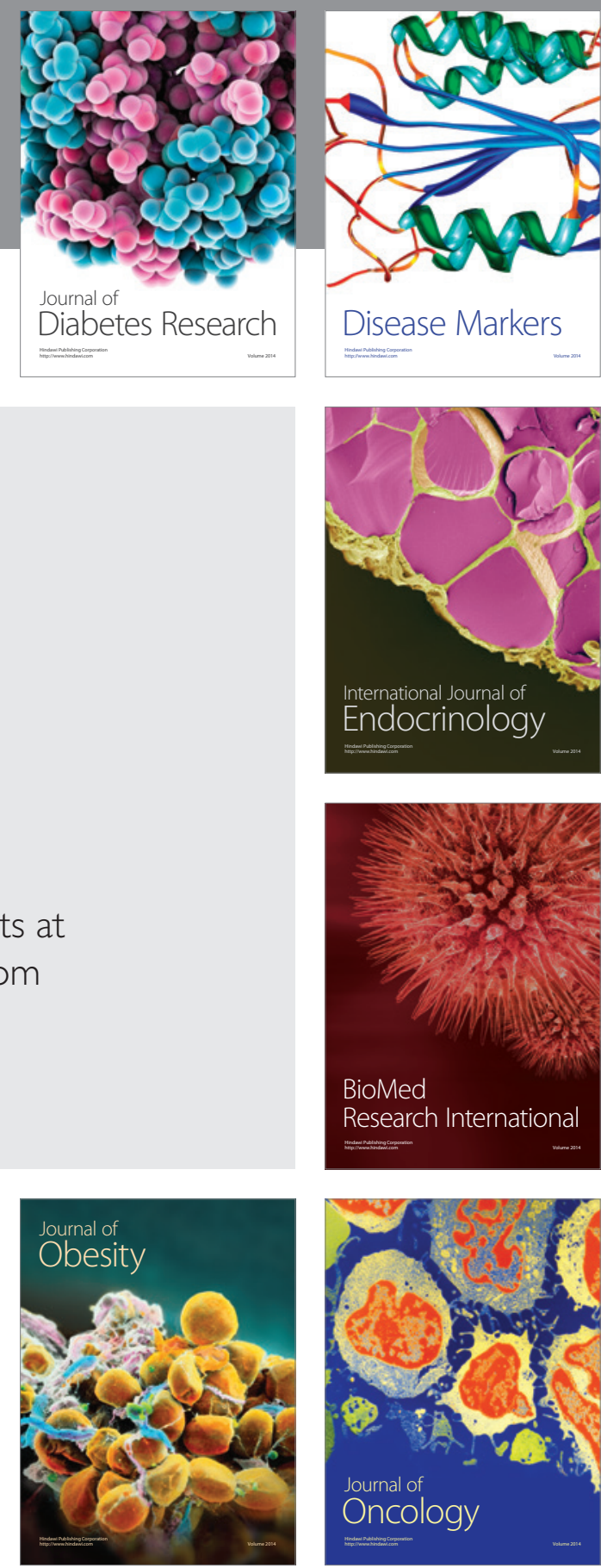

Disease Markers
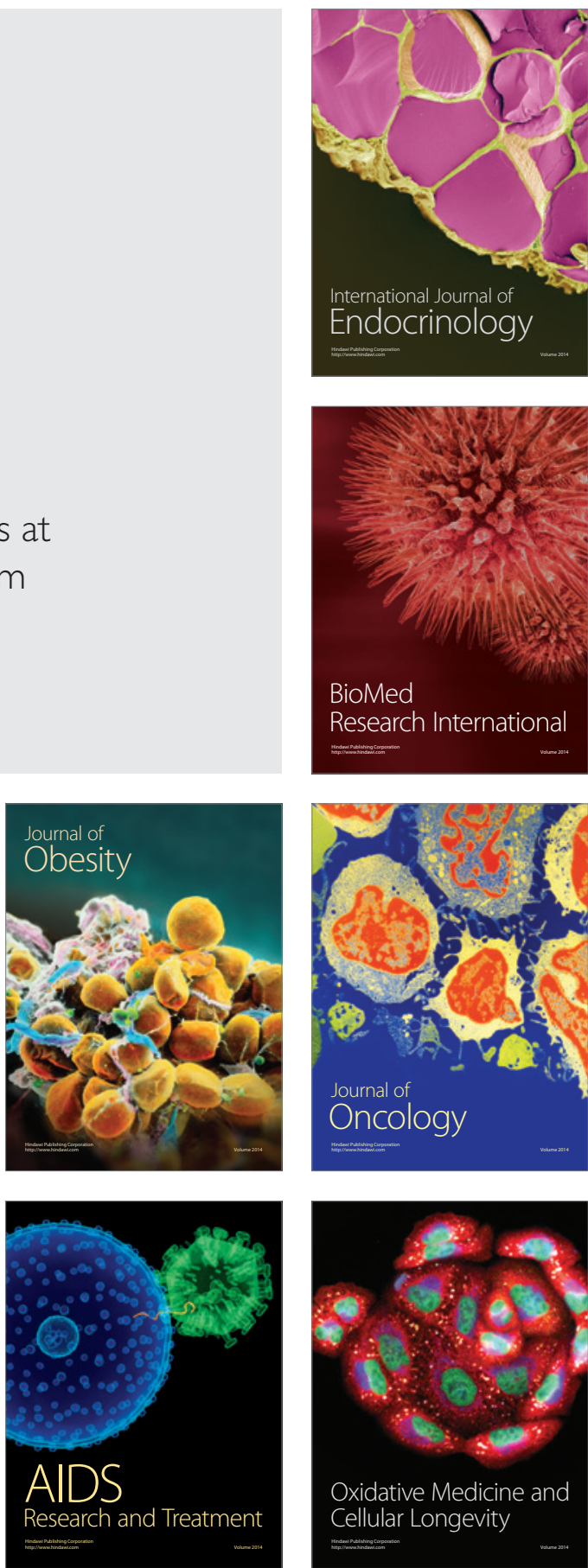